Commun. math. Phys. 35, 101-112 (1974)

(C) by Springer-Verlag 1974

\title{
The Yang-Lee Distribution for a Class of Lattice Gases
}

\author{
John S. N. Elvey \\ Mathematics Department, University of Otago, Dunedin, New Zealand
}

Received August 30, 1972; in revised form June 1, 1973

\begin{abstract}
Classical lattice gases moving on a simple cubic lattice are considered. The lattice is assumed to grow only one-dimensionally. The gas particles have hard cores (of diameter greater than the lattice spacing) and are further subject to interactions of finite range and finite order. The interactions outside the hard cores may be represented as the components of a $v$-dimensional vector, $\varphi$, which is initially allowed to be complex.

Using a transfer matrix technique, an asymptotic expression is obtained for the grand canonical pressure (at complex values of the inverse absolute temperature $\beta$ and the fugacity $z$ ).

Let $\lambda_{1} \ldots \lambda_{M}$ denote the eigenvalues of the transfer matrix. Define $\varphi$ to be a $D^{*}$-interaction if and only if the quotients, $\lambda_{J} / \lambda_{k}, 1 \leqq j<k \leqq M$, regarded as functions of $\beta, z$ (with $\varphi$ fixed) are nonconstant. In this paper it is assumed that there exists at least one allowable $D^{*}$-interaction. With this assumption, the main result is that if $F$ denotes the set of interaction vectors for which the distribution, $\Omega$, of limit points of zeros of the grand partition function in the complex $z$-plane at fixed $\beta$ (res. complex $\beta$-plane at fixed $z$ ) contains a domain, then $F$ contains no product set $A_{1} \times \cdots \times A_{v}, A_{k} \subset \mathbb{C}, 1 \leqq k \leqq v$ unless one or more of the $A_{k}$ consists of (at most) isolated points. This implies that the set of vectors for which $\Omega$ consists of arcs is dense in the set of all allowable vectors (in the usual topology for $\mathbb{C}^{v}$ ).
\end{abstract}

\section{Introduction}

Since Yang and Lee [1] formulated their theory of phase transitions and proved the celebrated "Circle Theorem", for ferromagnetic Ising models, much work has been done to obtain more general results. The greatest advance has been the extension of the circle theorem to quite general classes of "quasi-ferromagnetic" Ising and Heisenberg models, including those with suitable many-spin interactions [2].

The principles of the Yang-Lee theory apply to any statistical mechanical system. In all cases the physical behaviour of the model depends on the precise form of the distribution, say $\Omega$, of limit points of zeros, in the complex plane, of the grand partition function [3].

For the quasi-ferromagnets, the circle theorem states (in effect) that $\Omega$ is a subset of the unit circle; so that $\Omega$ contains at most one positive 
element. However, it has been shown recently [4] that the circle theorem breaks down at high temperatures if many-spin interactions are present in the model. Moreover, it is possible for $\Omega$ to fill the whole complex plane, as an example has shown ${ }^{1}$.

The Yang-Lee results and their extensions apply directly to the lattice-gas analogues of the magnetic models; but it is natural, for lattice gases, to allow: (i) gas particles with effective hard cores (leading to exclusion effects near occupied sites), and (ii) finite repulsive forces as well as attraction, outside the hard cores. For such models as these, very few rigorous results are known about $\Omega$. In particular, the circle theorem is invalid ${ }^{2}$ and it is by no means clear even that $\Omega$ must consist of "curves" rather than "regions" in the complex plane. In the case of continuous models the mathematical difficulties are very basic since $\Omega$ depends on detailed analytic properties of the grand partition function. Nevertheless, on the basis of a general conjecture due to Penrose (unpublished) it has been possible [5] to determine $\Omega$ for a class of onedimensional continuum models.

The purpose of my work is to show that, subject to one more condition, the conjecture holds also "for almost all ${ }^{3}$ interactions" for a class of lattice gases including those where effective hard cores and many-body (attractive or repulsive) forces of finite order and finite range are present.

To formulate this extra condition, we define an interaction vector $\varphi$ to be a $D^{*}$-interaction if and only if all of the quotients $\lambda_{j} / \lambda_{k}(j \neq k)$ of eigenvalues of the transfer matrix (regarded as functions of $\beta$ and $z$, with $\varphi$ fixed) are nonconstant. The main result of this paper may now be formulated as follows.

Let $\Phi$ denote the set of all allowable interactions (see Section 2). Define $F \subset \Phi$ by $F=\left\{\varphi \in \Phi: \Omega_{\varphi}\right.$ contains a domain $\}$, where $\Omega_{\varphi}$ denotes the Yang-Lee distribution corresponding to the given interaction $\varphi$.

If $\Phi$ contains at least one $D^{*}$-interaction then $\Phi \backslash F$ is dense in $\Phi$, in the standard topology for $\mathbb{C}^{\nu}$. In fact, every real vector in $\Phi$ is a limit point of real vectors in $\Phi \backslash F$.

Roughly speaking, this means that the Yang-Lee distribution consists of arcs "for almost all interactions" in this class of models. This will be proved in Propositions 4, 5, and 6. The formal definition of $\Omega$ is as follows.

\footnotetext{
${ }^{1}$ See [4], Theorem 3.

2 See for instance Hemmer, P.C., Hauge, E.H., Aasen, J. O.: J. Math. Phys. 7, 35 (1966).

A recent result of Ruelle [Phys. Rev. Lett. 26, 303 (1971)] shows that when repulsive forces (but only "point hard cores") are allowed for lattices with periodic boundary conditions then $\Omega \subset \mathscr{D}$ where $\mathscr{D}$ is a bounded region which may, at sufficiently low temperatures, include a segment of the positive real axis in the $z$-plane.

${ }^{3}$ This is not quite the standard use of the term "almost all": it is stronger (see Proposition 6).
} 
Let $\left\{Q_{r}(w)\right\}$ denote the sequence of grand partition functions (where $w$ stands for either of $\beta, z$ at a fixed value of the other).

Then we define $\Omega$ by the condition ${ }^{4}$ :

$$
w \in \Omega \Leftrightarrow \forall \eta>0 \forall t>0 \exists \omega \in \mathscr{N}_{\eta}(w) \exists r>t: Q_{r}(\omega)=0 .
$$

\section{Specification of the Models}

We consider a simple cubic lattice growing only one-dimensionally and with cyclic boundary conditions in the direction of growth. The finiteness of the range and order of the forces make it possible to specify the interactions as the components of a vector, say $\psi$, in $\mathbb{C}^{v+1}$, where $v$ is independent of the variable length of the lattice. We shall also suppose that the one-body forces are constant over the lattice (constant external field) with value $\mu$, so that we may write $\psi$ as an ordered pair $(\mu, \varphi)$, where $\varphi$ specifies all other interactions outside the hard cores and hence $\varphi \in \mathbb{C}^{\nu}$. We now define the class of vectors $\varphi$ more precisely.

Since the cross-section, say $\mathbb{D}\left(\subset \mathbb{Z}^{2}\right)$, is fixed, we may denote a general chunk of lattice of length $r$ by $L(r)$. Let $R=\operatorname{Max}\left(R_{k}: 1 \leqq k \leqq K\right)$ where $R_{k}$ denotes the finite range of the $k$-body forces. For any subset $A$ of $\mathbb{Z}^{d}$, denote by $\delta(A)$ the diameter of $A$ and by $\mathscr{P}^{*}(A)$ the set of all subsets of $A$ at the points of which gas particles could be placed with no hard cores overlapping.

Now define a function $\varphi$ on $\mathscr{P} *(\mathbb{D} \times \mathbb{Z})$ by: $\varphi: \mathscr{P}^{*}(\mathbb{D} \times \mathbb{Z}) \rightarrow \mathbb{C}$; $Y \mapsto \varphi_{Y}$, where

(a) $\varphi_{Y+j \hat{e}}=\varphi_{Y}$ (Translational invariance in the direction, $\hat{e}$, of growth of the lattice).

(b) $\varphi_{Y}=0$ if $\delta(Y)>R$ (finite total range of interactions).

In view of conditions (a) and (b), the function $\varphi$ may be replaced for purposes of calculation by its restriction to the finite set $\mathbb{D} \times\{1,2, \ldots, R\}$. This restriction may be associated with a vector in $\mathbb{C}^{v}$, where $v<2^{D R}$, $D$ being the number of points in $\mathbb{D}$.

\section{The Transfer Matrix}

All of the subsequent analysis is based on the representation of the grand partition function as a trace ${ }^{5}$.

${ }^{4} \mathcal{N}_{\eta}(w)=\left\{w^{\prime}:\left|w-w^{\prime}\right|<\eta\right\}$.

5 This general formalism is well-known, so the details are omitted. For a detailed treatment for strictly one-dimensional models see Baur, M.E., Nosanow, L.S.: J. Chem. Phys. 37, 153 (1962), Appendix. 
Proposition 1. There exists a square matrix $T$ of finite order $M$, independent of $r$, such that

$$
Q_{r}(\psi) \equiv \sum_{Y \in \mathscr{P}^{*}(L(r))} \prod_{X \subset Y} e^{-\beta \psi_{X}}=\operatorname{tr}\left[T^{r}(\psi)\right]
$$

Proof. Let $C_{k}$ denote the difference of the sets $L(k+R-1)$ and $L(k)$, where $R$ is the overall range of the interaction and $C_{r+k} \equiv C_{k}$.

Let $U \subset C_{k}$ and $V \subset C_{k+1}$.

Define a relation $\approx$ (with negation $\approx$ ) by:

$$
U \approx V \Leftrightarrow\left[U \cup V \subset \mathscr{P}^{*}(L(r)) \wedge U \cap C_{k+1}=V \cap C_{k}\right] .
$$

Then $T$ may be specified as:

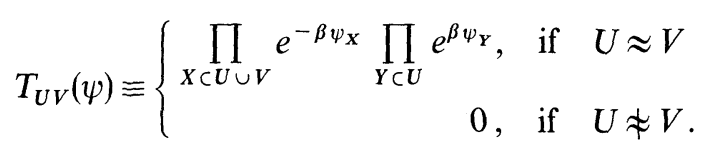

The cyclic boundary conditions on $L(r)$ give the trace of $T^{r}$, as required.

\section{Characterization of $\boldsymbol{\Omega}$ in Terms of the Function $\Lambda$}

So defined, $T$ is a matrix-valued analytic function with domain $\mathbb{C}^{2} \times \Phi$, corresponding to the variables $\beta, z$ and $\varphi$. For the moment it is convenient to regard $\varphi$ as fixed in $\Phi$ and to write $w$ for either of the variables $\beta, z$ at a fixed value of the other. The function $T$ may then be regarded as an analytic matrix-valued function of the single variable $w$.

Let us denote by $A$ the analytic function determined by the characteristic equation of $T$ (which is a polynomial equation, $P_{T}(\lambda)=0$, where all coefficients are entire functions of $w$ ). We now define subsets $S_{1}, S_{2}$, and $S$ of $\mathbb{C}$ as follows:

$$
\begin{aligned}
& S_{1} \equiv\{w \in \mathbb{C}: \Lambda \text { has a maximodular branch point at } w\} ; \\
& S_{2} \equiv\{w \in \mathbb{C}: \Lambda \text { has no maxi-modular branch point at } w \\
& \text { but at least two maximodular branches }\} ; \\
& S \equiv S_{1} \cup S_{2} .
\end{aligned}
$$

Proposition 2. (a) $S_{1} \subset \bar{S}_{2}$,

(b) $S$ is closed in $\mathbb{C}$,

(c) $\Omega \subset S$.

Proof. (a) The form of $P_{T}$ shows ${ }^{6}$ that $\Lambda$ has at most $M$ values for each value of $w$, and that the branch points of $\Lambda$ are isolated and all

${ }^{6}$ See, for instance, Saks, S., Zygmund, A.: “Analytic Functions”, Chapter VI, $\$ 14-15$ (Warszaw 1965, P.W.N. Scientific Publications). 
algebraic. Let $\alpha \in S_{1}$ be a branch point of order $q-1$ at which $q$ branches, say $\lambda_{1}, \ldots, \lambda_{q}$, of $\Lambda$, assume the common, maximodular value $\beta$.

Then there is a deleted neighbourhood of $w=\alpha$, say $P_{\delta}(\alpha)$, on which

$$
\lambda_{k}(w)=\beta+\sum_{l=1}^{\infty} A_{k l}\left[(w-\alpha)^{1 / q}\right]^{l}
$$

for $1 \leqq k \leqq q$, where the $A_{k l}$ are complex coefficients and $(w-\alpha)^{1 / q}$ denotes a definite value (the same for each $k$ ) determined from the equations (in $\theta): \theta^{q}=w-\alpha(w \neq \alpha)$. Consider now the functions $g_{k}$, where, for $1 \leqq k \leqq q$,

$$
g_{k}(\zeta) \equiv \beta+\sum_{l=1}^{\infty} A_{k l} \zeta^{l} .
$$

The $g_{k}$ are regular on $\mathscr{N}_{\delta^{1 / q}}(0)$, and satisfy, on $P_{\delta}(\alpha)$ the conditions:

$$
g_{k}\left[(w-\alpha)^{1 / q}\right]=\lambda_{k}(w) .
$$

Since the branches $\lambda_{1} \ldots \lambda_{q}$ assume at $w=\alpha$ a common maximodular value, the quotients $F_{m n}(\zeta) \equiv g_{m}(\zeta) / g_{n}(\zeta)$ are also regular on $\mathscr{N}_{\delta^{1 / q}}(0)$ and, moreover, $\left|F_{m n}(0)\right|=1$ for $1 \leqq m<n \leqq q$.

By applying the Maximum Modulus theorem separately to $F_{m n}$ and to $1 / F_{m n}$ on $\mathscr{N}_{\delta^{1 / q}}(0)$, we conclude that 1 cannot be either the maximum or the minimum value of $\left|F_{m n}\right|$ on (say) $\overline{\mathcal{N}_{\frac{1}{2}} \delta^{1 / q}(0)}$. We may now deduce, from the conditions: $g_{k}\left((w-\alpha)^{1 / q}\right)=\lambda_{k}(w)$ on $P_{\delta}(\alpha)$, that every neighbourhood of $w=\alpha$ contains a point of $S_{2}$, and hence that $S_{1} \subset \bar{S}_{2}$, as asserted.

(b) To prove that $S_{1} \cup S_{2}$ is closed in $\mathbb{C}$, it is sufficient to show that the complement of $S_{1} \cup S_{2}$ is open. However, at a point $w^{\prime}$ of the complement of $S_{1} \cup S_{2}$, the analytic function $\Lambda$ has a unique maximodular branch, and this branch is regular. Such a situation plainly obtains at the points of some neighbourhood of $w^{\prime}$; and hence, $S_{1} \cup S_{2}$, as the complement in $\mathbb{C}$ of an open set, is itself a closed set.

To prove (c) we observe that if $w \in \mathbb{C} \backslash S$ then $\Lambda$ has a unique maximodular branch (say $\lambda_{1}$ ) and this branch is regular in a neighbourhood, say $\mathscr{N}(w)$, of $w$.

Using the trace representation of $Q_{r}$ we now obtain:

$$
Q_{r}(w)=\lambda_{1}^{r}(w)\left[1+\sum_{2 \leqq j \leqq M}\left\{\lambda_{j}(w) / \lambda_{1}(w)\right\}^{r}\right]
$$

so that, if $\mathscr{D}$ is any closed subdisc of $\mathscr{N}(w)$, then

$$
Q_{r}(w)=\lambda_{1}^{r}(w)[1+o(1)]
$$

where $o(1)$ tends uniformly to 0 on $\mathscr{D}$ as $r$ tends to $\infty$. Hence $\mathscr{D}$ contains no zeros of $Q_{r}$ for all sufficiently large values of $r$, so that, since $w \in \mathbb{C} \backslash S$ $\Rightarrow w \in \mathbb{C} \backslash \Omega$, we have $\Omega \subset S$, as required. 
We show next that if $S$ contains no domain then $S$ is a subset of $\Omega$, which implies that $\Omega$ coincides with $S$. The Yang-Lee set is thus characterized in terms of a criterion depending only on the interaction vector.

Proposition 3. If $S$ contains no domain then $\Omega$ coincides with $S$.

Proof $^{7}$. In view of Proposition 2(c) it is enough to show that $\Omega \supset S$. Since $\Omega$ is closed (by definition of "limit point of zeros") and $S_{1} \subset \bar{S}_{2}$ [by Proposition 2(a)] we need only show that $\Omega \supset S_{2}$.

Suppose not, that is, suppose that $w_{0} \in S_{2} \backslash \Omega$. Then there is a neighbourhood, say $\mathscr{N}\left(w_{0}\right)$, containing no zeros of the functions $Q_{r}$ for all sufficiently large $r$, and hence a regular branch of $Q_{r}^{1 / r}$ may be defined on $\mathcal{N}\left(w_{0}\right)$ for all sufficiently large $r$. On writing $Q_{r}(\psi)$ explicitly in terms of $\beta$ and $z$ one has:

so that

$$
\left|Q_{r}(w)\right|=\left|\sum_{k=1}^{M}\left\{\lambda_{k}(w)\right\}^{r}\right|
$$

$$
\left|\left[Q_{r}(w)\right]^{1 / r}\right| \leqq M^{1 / r} \operatorname{Max}_{1 \leqq k \leqq M}\left|\lambda_{k}(w)\right|
$$

(It is worth noting that a uniform upper bound on $Q_{r}^{1 / r}(w)$ can always be found for a system with stable potential - see, for instance, Ruelle ${ }^{11}$ ).

It follows that the sequence $\left\{Q_{r}^{1 / r}(w)\right\}$ is uniformly bounded on each closed subdisc, $\mathscr{D}$, of $\mathscr{N}\left(w_{0}\right)$. Hence, by a corollary [6] to the Vitali convergence theorem, there is a subsequence, say $\left\{Q_{r_{j}}^{1 / r_{J}}\right\}$, uniformly convergent on $\mathscr{D}$ to a limit function $q$, where $q$ is regular over $\mathscr{D}$. Since, by hypothesis, $S$ contains no domain, one may, without loss of generality, assume that $q\left(w^{\prime}\right)=\lambda_{1}\left(w^{\prime}\right)$ at some point $w^{\prime} \in \mathscr{D}$, and hence throughout $\mathscr{D}$. On the other hand, since $\mathscr{D}$ contains points of $S_{2}$, there are certainly points $w^{\prime \prime} \in \mathscr{D}$ where $\lambda_{1}$ is not the unique maximodular branch, and at such points $\left\{Q_{r_{j}}^{1 / r_{j}}\left(w^{\prime \prime}\right)\right\}$ cannot tend to $\lambda_{1}\left(w^{\prime \prime}\right)$. This contradiction proves the theorem.

We prove now ${ }^{8}$ that if $S$ contains no domain, then $S$ (and hence $\Omega$ ) consists of analytic arcs.

Proposition 4. If $S$ contains no domain then $\Omega$ comprises a system of closed analytic arcs.

${ }^{7} \mathrm{I}$ am indebted to an anonymous referee for this elegant indirect proof, which is both simpler and more widely valid than the constructive proof (cf. Ref. [7] §6) that I had intended to use. However, one result is lost in the present scheme; namely, that the density on $\Omega$ equals $(1 / 2 \pi)(\partial / \partial \alpha) \Delta_{\sigma} \log \lambda_{\max }(w)$, where $\Delta_{\sigma} \log \lambda_{\max }(w)$ is the discontinuity in the imaginary part of the pressure "across $\Omega$ at $w(\sigma)$ ", and each zero of $Q_{r}$ is given the weight $r^{-1}$. Here $\sigma$ denotes a real parameter for the "curves" $\Omega$.

8 Cf. Ref. [5] §5. 
Proof. In view of Proposition 3 it suffices to prove that $S$ has an analytic parametrization with a real parameter.

Let $w \in S_{2}$. Then, in a sufficiently small neighbourhood, $\mathscr{N}(w)$, there are branches, say $\lambda_{p_{l}} \ldots \lambda_{p_{1}}$, such that for $1 \leqq a<b \leqq l$, we have

$$
w \in S_{2} \cap \mathcal{N}(w) \Rightarrow \lambda_{p_{a}}(w) / \lambda_{p_{b}}(w)=\exp \left\{i v_{a b}\right\}
$$

where the numbers $v_{a b}$ are real.

Now the quotient $\gamma_{a b} \equiv \lambda_{p_{a}} / \lambda_{p_{b}}$ is regular (by definition of $S_{2}$ ) and nonconstant (since $S$ contains no domain, by hypothesis). Hence $\gamma_{a b}$ has nonvanishing derivative except (at most) on a set of isolated points.

Consequently, the inverse function $\gamma_{a b}^{-}$is well defined on $S \cap \mathcal{N}(w)$, except (possibly) at isolated points. The set $S$ may be parametrized through a finite collection of representations of form:

$$
S_{a b}=\left\{w: w=g_{a b}\left(v_{a b}\right)\right\}
$$

where $g_{a b}\left(v_{a b}\right) \equiv \gamma_{a b}^{-1}\left[\exp \left\{i v_{a b}\right\}\right]$.

The closure of this system of analytic arcs coincides with $\Omega$. We show now that the set $F \equiv\{\varphi \in \Phi: S$ contains a domain $\}$ is "small" in a certain sense. First we need the following result.

Proposition 5. If $S$ contains a domain then $\Lambda$ has two branches that are everywhere equimodular.

Proof. Let $\lambda_{1}$ and $\lambda_{2}$ be maximodular (and hence equimodular) on a domain $\mathscr{D} C S$. Then, as in the proof of Proposition 4, the quotient $\lambda_{1} / \lambda_{2}$ is regular on $\mathscr{D}$, but in this case its logarithm (which is also regular since $\mathscr{D}$ may be chosen so that $\lambda_{1} \neq 0, \lambda_{2} \neq 0$ on $\left.\mathscr{D}\right)$ has constant real part, namely zero, on $\mathscr{D}$.

By the Cauchy-Riemann conditions, therefore, $\operatorname{Im}\left\{\log \left[\lambda_{1} / \lambda_{2}\right]\right\}$ is constant over $\mathscr{D}$, whence it follows that $\lambda_{1}(w)=\lambda_{2}(w) \exp \{i t\}$ for some fixed $t \in \mathbb{R}$ and all $w \in \mathscr{D}$; which implies that this relation holds for all values of $w$, with the same fixed value of $t$.

\section{Proof that $F$ is "Small"}

For our purposes it is better to state Proposition 5 in the equivalent form:

Proposition 5'. If $A$ does not have (at least) two branches everywhere equimodular then $S$ contains no domain.

The next (crucial) proposition shows that the set of vectors $\varphi$ for which $\Lambda$ has at least two everywhere-equimodular branches is "small" provided that $\Phi$ contains at least one $D^{*}$-interaction. 
Proposition 6. Assume that $\Phi$ contains a $D^{*}$-interaction and let

$$
F \equiv\{\varphi \in \Phi: S \text { contains a domain }\}
$$

If $A_{1} \times A_{2} \times \cdots \times A_{v} \subset F$ where each $A_{k} \subset \mathbb{C}$, then at least one of the $A_{k}$ contains no point of accumulation.

Proof. We shall prove Proposition 6 as a corollary of the following stronger result:

Proposition 6'. The result in Proposition 6 holds also for the set $F^{\prime}$ defined by:

$$
F^{\prime} \equiv\{\varphi \in \Phi: \Lambda \text { has two branches in a constant ratio }\} .
$$

We observe first that $\varphi \in F^{\prime}$ if and only if there is a number $s \in \mathbb{C}$, independent of $w$, such that, regarded as polynomials in $\lambda$, the polynomials $P_{T}(\lambda)$ and $P_{T}(\lambda s)$ have (for all $\left.w\right)$ a common zero. This, in turn, occurs if and only if the resultant in $\lambda$ of $P_{T}(\lambda)$ and $P_{T}(\lambda s)$ vanishes identically in $w$. Next, we note that, from the definition of $T$, all matrix elements of $T$ have the form

$$
T_{k l}=z^{\alpha_{k l}} \exp \left\{\beta \sum_{1 \leqq m \leqq \nu} B_{k l m} \varphi_{m}\right\}
$$

where the $\alpha_{k l}$ and $B_{k l m}$ are integers and the $\alpha_{k l}$ are nonnegative, and $\varphi_{1} \ldots \varphi_{v}$ are the components of a vector $\varphi$. Moreover, since both $P_{T}$ and the resultant of $P_{T}(\lambda)$ and $P_{T}(\lambda s)$ are determinants, it follows that, as a polynomial in $s$, this resultant, say $\varrho(s)$, has coefficients of form

$$
d_{k}=\sum_{1 \leqq i \leqq M_{k}} z^{a_{i}} \exp \left\{\beta \sum_{1 \leqq j \leqq v} G_{i j k} \varphi_{j}\right\}
$$

where $a_{i} \in \mathbb{N}$ and $G_{i j k} \in \mathbb{Z}$.

Thus the $d_{k}$ are entire functions of $\beta, z$ and each component of $\varphi$, and hence $d_{k}$ has an everywhere-convergent series expansion in any or all of these variables, the convergence being absolute and uniform on any relevant compact set. Since ${ }^{9}$ arbitrary rearrangements of an absolutely convergent multiple power series leave its sum unchanged, we may now assert that $\varrho$ has an everywhere-valid representation in the form

$$
\varrho(\varphi, w, s)=\sum_{j \in \mathbb{N}} \sum_{1 \leqq k \leqq H} \delta_{j k}(\varphi) w^{j} s^{k}
$$

where $\mathbb{N}$ is the set of natural numbers and $H$ is a finite integer, depending on the overall range of the interactions. We conclude that $\varrho$ vanishes

\footnotetext{
${ }^{9}$ See, for example [6], $\S \S 1.62-1.64$, from which our assertion follows directly.
} 
identically in $w$ only if $\varphi$ satisfies the sequence of conditions:

$$
q_{j}(s) \equiv \sum_{1 \leqq k \leqq H} \delta_{j k}(\varphi) s^{k}=0 \quad(j \in \mathbb{N})
$$

for some fixed value of $s$, the coefficients $\delta_{j k}(\varphi)$ being entire functions of $\varphi$.

A necessary condition for the existence of a common zero of all the $q_{j}$ is that, for the vector $\varphi$ in question, all of the resultants $\varrho_{a b}$ of distinct polynomials $q_{a}, q_{b}$ should vanish. The set of values of $\varphi$ for which this can occur is, plainly, a subset of the null set of any one of these resultants.

We observe now that the existence of a $D^{*}$-interaction implies that not all of the $\varrho_{a b}$ vanish identically on $\Phi$. Let $\varrho_{m n} \neq 0$.

We deduce that

$$
F^{\prime} \subset\left\{\varphi \in \Phi: \varrho_{m n}(\varphi)=0\right\} .
$$

We are now in a position to complete the proof of Proposition 6.

Since $\varrho_{m n}$ is just a determinant involving the coefficients of $q_{m}$ and $q_{n}$, which are entire functions of each component of $\varphi$, the condition $\varrho_{m n}(\varphi)=0$ exhibits $\varphi$ as a zero of an entire function.

Since the $\varrho_{m n}$ are continuous in $\varphi$, the null set of $\varrho_{m n}$ is closed. Moreover, if we write $\varrho_{m n}$ as an (everywhere-convergent) multiple power series, then the basic condition has form

$$
\sum_{k_{1}, \ldots, k_{v} \in \mathbb{N}} a_{k_{1} \ldots k_{v}} \varphi_{1}^{k_{1}} \ldots \varphi_{v}^{k_{v}}=0
$$

It is a standard result from the theory of functions of several complex variables that the null set defined by this condition is closed and nowheredense in $\mathbb{C}^{\nu}$. For our purposes, however, a slightly stronger condition is desirable, namely the condition in Proposition 6'. This may be established by repeated applications of the identity theorem for power series in a single variable ${ }^{10}$, starting with

$$
\sum_{k_{1} \in \mathbb{N}}\left(\sum_{k_{2} \ldots k_{v} \in \mathbb{N}} a_{k_{1} k_{2} \ldots k_{v}} \varphi_{2}{ }^{k_{2}} \ldots \varphi_{v}{ }^{k_{v}}\right) \varphi_{1}{ }^{k_{1}},
$$

which shows, after $v$ steps, that if $F^{\prime}$ contains a product set of form $A_{1} \times A_{2} \times \cdots \times A_{v}, A_{k} \subset \mathbb{C}, 1 \leqq k \leqq v$, where each $A_{k}$ has at least one point of accumulation, then all of the coefficients $a_{k_{1} \ldots k_{v}}$ vanish. Since $F^{\prime} \subset \Phi$ and $F \subset F^{\prime}$, the proof is complete.

Corollary. Let $\varphi \in \Phi \cap[\mathbb{R} \times\{0\}]^{\nu} \equiv \Phi_{\mathbb{R}}$ and let $\varepsilon$ be an arbitrary positive number.

Then there is at least one vector $\varphi^{*}$ in $\Phi_{\mathbb{R}}$ such that

(a) $\varphi^{*} \notin F$

(b) $\left\|\varphi-\varphi^{*}\right\| \equiv N\left\{\sum_{1 \leqq k \leqq \nu}\left(\varphi_{k}-\varphi_{k}^{*}\right)^{2}\right\}<\varepsilon$.

${ }^{10}$ See, for instance, [6], p. $87, \S 2.6$. 
Proof. Suppose that the Corollary is false. Then $F^{\prime}$ contains a product set $A_{1} \times \cdots \times A_{v}$, where

$$
A_{k} \equiv\left\{\varphi_{k} \in \mathbb{R}:\left|\varphi_{k}-\varphi_{k}^{*}\right|<\frac{\varepsilon}{v}\right\}
$$

which contradicts Proposition 6'.

\section{Discussion}

In the foregoing work, the following basic assumptions have been made.

(a) $\Phi$ contains a $D^{*}$-interaction.

(b) The lattice grows only one-dimensionally.

(c) The interactions have finite range and finite order.

(d) The interactions are translationally invariant (at least in the direction of growth).

(e) Cyclic boundary conditions are imposed.

The basic assumption (a), that $\Phi$ contains at least one $D^{*}$-interaction, is of a purely technical nature. Hopefully, it can eventually be removed by the construction of a $D^{*}$-interaction, but this has not, so far, been achieved. When there are no effective hard cores, assumptions (b) and (c) preclude the existence of a phase transition in the strictly mathematical sense ${ }^{11}$. This limitation can be overcome only if a suitable asymptotic formula can be found for the grand partition function when one (or both) of conditions (b) and (c) is removed.

When the hard cores can overlap the situation is not so clear-cut. [See, for instance, Nilsen, T.S., Hemmer, P.C.: J. Chem. Phys. 46, 2640 (1967)]. The general result is still an open question.

The translational invariance condition is commonly imposed and is physically acceptable in a wide variety of situations. The cyclic boundary condition, however, could, ostensibly, affect the equation of state.

That this is not so in the present models follows ${ }^{12}$ from conditions (b) and (c). For, let both of $\beta, z$ be positive. Since the cross-section, $\mathbb{D}$, of the lattice is fixed and finite, the extra interactions introduced by imposing cyclic boundary conditions contribute an additional factor less than $\exp \left\{2^{M_{0}} \beta J\right\}$ to each term in the "grand partition sum", where $M_{0}$ is the maximal number of nonoverlapping particles that can be found in a chunk of lattice having cross-section $\mathbb{D}$ and length $2 R$ (twice the overall range of interaction) and $J \equiv \operatorname{Max}\left\{\left|\varphi_{X}\right|: X \in \mathscr{P}^{*}(L(R))\right\}$. Consequently, if $h_{r}(\beta, z)$ denotes the difference between the "cyclic" and

${ }^{11}$ See, for instance, Ruelle, D.: Statistical mechanics, Sec. 5.6. (New York: W. A. Benjamin Inc. 1969). See also Baur and Nosanow, footnote 5.

12 The results on classical continuum systems in a toroidal container obtained by Fisher and Lebowitz (Commun. math. Phys. 19, 251 (1970)) probably extend easily to lattice systems, but the proof given here is simple and self-contained. 
"free" pressures for a lattice of length $r$, then we have

$$
\left|h_{r}(\beta, z)\right|=r^{-1}\left|\log \left[Q_{r}^{\text {cyclic }}(\beta, z) / Q_{r}^{\text {free }}(\beta, z)\right]\right|<2^{M_{0}} \beta J / r
$$

for positive values of $\beta$ and $z$.

Hence $h_{r}(\beta, z)$ tends uniformly to 0 as $r$ tends to $\infty$, so that the operations $r \rightarrow \infty$ and $\partial / \partial \beta, \partial / \partial z$ may be interchanged, leaving the equation of state unaffected by the boundary condition.

On a more practical note, it has been shown recently [7] that if $\Omega$ is assumed to consist of arcs in the $\beta$-plane, then all of the standard "critical behaviour" can be obtained by suitable choice of the density of limit points of zeros on $\Omega$. Indeed, it is possible to obtain physically interesting results by supposing that $\Omega$ does contain a domain (whose intersection with the positive real axis in the $\beta$-plane must, of course, consist of isolated points, at most) and then prescribing a particular form for the density on this domain. Similar work [8] has been done in terms of assumed forms of density on $\Omega$ in the $z$-plane. Here, however, for Ferromagnetic systems, the Lee-Yang Circle Theorem guarantees that $\Omega$ contains no domain ${ }^{13}$. It is clear from the present work that only the maximodular eigen-values of $T$ are relevant to the equation of state (though it is known that the other eigen-values can be related to various correlation functions). There seems, however, to be no simple criterion for identifying eigen-values of co-maximal moduli rather than merely equal moduli. (Criteria involving differentiation in $\lambda$ would not improve the results.)

Acknowledgements. I thank O. Penrose for advice in the initial stages of this work. An earlier version of this paper was written during 1969 in Leiden, as part of the research program of the Stichting Voor Fundamenteel Onderzoek der Materie (F.O.M.), with a grant from the Netherlands Organization for Pure Scientific Research (Z.W.O.). It is a pleasure to thank Professor P. Mazur for his kind hospitality at the Lorentz Institute, and to thank C. M. Fortuin (Leiden) and D. G. B. Hill (Otago) for helpful discussions. Most of all, I am indebted to A. van de Ven for drawing my attention to the relevance of analytic sets to the proof of Proposition 6, and to the anonymous referee for constructive criticism, as well as for the alternative proof of Proposition 3.

\section{References}

1. Yang, C. N., Lee, T.D.: Phys. Rev. 87, 404 (1952)

Lee, T. D., Yang, C. N.: Phys. Rev. 87, 410 (1952)

2. Suzuki, M., Fischer, M.E.: J. Math. Phys. 12, 235 (1971)

See also Asano, T. Progr. Theoret. Phys. (Kyoto) 40, 1328 (1968), J. Phys. Soc. 25, 1220 (1968); 29, 350 (1970)

13 For the two-dimensional ferromagnetic Ising model, Fisher has shown that the distribution $\Omega$ in the complex plane of a suitable (hyperbolic) function of $\beta$, comprizes a pair of intersecting circles, one of which gives the known phase transition. (See Fisher, M. E., in Boulder Lectures in Theoretical Physics VII C 1964 (1965, U. of Colorado Press)). For Yang-Lee Theorems in the $\beta$-plane, see Jones, G. L.: J. Math. Phys. 7, 2000 (1966). 
3. See, for example, Huang, K., Statistical Mechanics, Chapter 15. New York: John Wiley \& Sons Inc., 1963

4. Heilmann, O. J.: J. Math. Phys. 11, 2701 (1970)

5. Penrose, O., Elvey, J. S. N.: J. Phys. A 1, 661 (1968)

6. See for instance, E.C.Titchmarsh, The Theory of Functions Second Edition, p. 169, Th. 5.22. Oxford 1939

7. Grossmann, S., Rosenhauer, W.: Z. Physik 207, 138 (1967)

8. Suzuki, M.: Prog. Theor. Physics 38, 1125 (1967)

John S. N. Elvey

c/o "Orchards", Summerhill Lane

Haywards Heath

Sussex, U.K.

Note Added in Proof. Methods closely related to the scheme considered here have been used by Katsura and Ohmanami (Progr. Theor. Phys., 1973). They deal mainly with Husimi-Temperley antiferromagnets. 